lation se trouve aux environs de $68^{\circ} \mathrm{C}$.; mais, cette valeur doit être considérée seulement comme approchée car sa détermination est incertaine. En effet, à mesure que la température de l'essai aug mente, le point de coagulation devient plus difficile à préciser ; dès $60^{\circ} \mathrm{C}$., les flocons se contractent, expulsant le sérum à l'instant de sa formation, une gélification nette n'ayant pas lieu. D'ailleurs, à des températures élevées, le lait emprésuré perd par évaporation une certaine quantité d'eau, modifiant sa concentration, ce qu'on ne peut pas empêcher en bouchant le récipient, puisque la vapeur qui se condense sur les parois forme une couche d'eau à la surface du lait emprésuré.

(A suivre.)

\title{
UNE TECHNIQUE SIMPLE PERIMETTANT L'ANALYSE COMPLÈTE DU LAIT DANS UN ÉCHANTILLON ASSEZ REDUIT DE CE LIQUIDE
}

\author{
par
}

\section{R. VLADESCO}

Professeur

Laboratoire de Chimie biologique,

Faculté de Módecine vétérinaire de Bucarest.

Le dosage des différents constituants du lait se fait ordinairement sur des prises d'essai isolées de ce liquide. Cela est possible toutes les fois que la quantité de lait dont on dispose est suffisamment grande.

Sans préjuger quant à la légitimité de eette manière de procéder, il est évident qu'elle présente des inconvénients sérieux lorsque ta quantité de lait disponible est réduite.

Cela, parce que, dans ce cas, on est obligé de recourir aux microméthodes dont l'application demande des soins multiples et minutieux, faute de quoi on est exposé à commettre des erreurs grossières.

Il est infiniment préférable, par conséquent, de séparer les constituants de la même prise d'essai du lait à analyser et de les doser ensuite. Des techniques remplissant ce desideratum ont été préconisées plusieurs fois (1) (2), et leur nombre doit être à coup sûr plus important que celui que nous connaissons.

La technique que nous proposons et dont les détails sont exposés dans cette note, sans être plus compliquée que celles sur lesquelles nous sommes documentés, présente l'avantage de permettre une analyse assez complète de lait.

(1) Penterin. Formulaire chimique des laboratoires modernes, 1927.

(2) Trifon Ugarte. Nouvelle méthode d'analyse du lait sur un centimètre cube. Annales des falsifications et des fraudes, nos 272-273, p. 402, 1931. 
Avec le désir de la voir contrôlée et surtout perfectionnée, parce qu'elle en est 'susceptible, nous nous permettons de la soumettre à l'attention des intéressés, en l'exposant avec tous les détails que nous avons eru nécessaires.

\section{LA DENSITE}

La densité peut être déterminée par la méthode du flacon, pourvu qu'on dispose de quelques centimètres cubes de lait, de flacons de grandeur convenable et d'une balance de précision.

\section{LE RÉSIDU SEC}

Dans une fiole pouvant être fermée hermétiquement, on pèse un papier à filtre sans cendres (1), après l'avoir préalablement desséché à l'étuve à $100^{\circ}-103^{\circ}$.

Sur ce morceau de papier appuyé sur le rebord d'un verre à pied, on répand uniformément un nombre déterminé de centimètres cubes de lait (ordinairement de 2 à 5 ).

Pour cela, on laisse s'écouler le lait, aspiré dans une pipette, goutte par goutte, sur la surface du papier (un seul côté). Lorsque le lait a été complètement absorbé, on enroule le papier en lui donnant la forme d'une eartouche à extraction. On laisse dessécher le papier à l'étuve à, $100^{\circ}-103^{\circ}$, le temps nécessaire, afin que l'eau s'évapore complètement. Une fois desséché, il est introduit dans la fiole avec laquelle il a été pesé au commencement.

Maintenue quelque temps dans un exicateur à chlorure de calcium, la fiole est de nouveau pesée.

L'excès de poids de cette pesée, sur celle du commencement, représente le résidu sec de la prise de lait mesurée en volumes.

\section{LA MATIËRE GRASSE}

Le papier enroulé, bien desséché, est introduit dans un appareil à extraction en se servant de l'éther sulfurique comme liquide extracteur. L'extraction faite, on dessèche le papier. Une nouvelle pesée, avec les précautions nécessaires, permet le dosage en bloc des lipides. En effet, la différence, entre cette pesée et la précédente, représente l'ensemble des matières solubles dans l'éther sulfurique, c'est-à-dire les graisses, les lécithines et les stérines.

\section{LE LACTOSE}

Le papier débarrassé de lipides est soumis ensuite à une extraetion ayant pour but la séparation du lactose et de toutes les autres matières solubles dans l'eau, des matières protéiques qui se trouvent dans le lait (caséine, albumine, globuline).

On arrive à cela en extrayant plusieurs fois (10-15) le papier intro-

(1) Le papier utilisé était fourni par la Maison Macherey, Nagel et Cie, Düren. 
duit dans un entonnoir à séparation, de forme cylindrique, avec robinet, avec une solution centième normale d'acide acétique. Le volume de la solution acétique utilisée est d'environ 100 fois plus grand que celui de la prise de lait. Les liquides séparés par décantation après chaque extraction sont recueillis dans une fiole jaugée.A la fin, on complète avec de l'eau distillée jusqu'à la marque.

L'extraction est faite à froid et sans trop de retard, l'expérience nous ayant montré que le lactose subit rapidement la fermentation lactique. Le liquide d'extraction contient le lactose, les sels solubles, l'acide citrique, les substances azotées solubles, etc.

Les substances protéiques du lait restent intégralement imprégnées dans le papier. (Dans le liquide séparé, nous n'en avons jamais trouvé par les réactifs habituels.)

Sur une portion déterminée de liquide renfermant environ $50 \mathrm{mgr}$. de lactose on peut doser ce corps par la méthode de Bertrand.

\section{LES GHLORURES}

Sur la portion restante de liquide on peut doser les chlorures par la méthode Charpentier-Volhard, à l'aide d'une solution dixième normale d'azotate d'argent.

Ce dosage est très facile lorsqu'il s'agit du lait de vache et lorsque la prise d'essai n'est pas inférieure à $2 \mathrm{~cm}^{3}$.

\section{LES PHOSPHATES SOLUBLES}

Toujours dans le même liquide on peut, assez commodément, doser les phosphates solubles.

Dans ce but, on introduit dans un creuset une portion déterminée du liquide séparé (généralement un dixième du volume total suffit). On y ajoute une dizaine de gouttes d'une solution d'acétate de magnésium à $20 \%$ et on évapore au bain-marie jusqu'à la siccité. Le résidu est ensuite incinéré, dans un four, jusqu'à l'obtention des cendres blanches. On dissoutles cendres àl'aide d'une solution d'acide sulfurique à $20 \%$ et, dans cette solution, on dose l'acide phosphorique.

\section{LES SELS SOLUBLES DE CALCIUM}

On évapore au bain-marie une portion de liquide de l'extraction précédente - soit un dixième du volume total - jusqu'à ce que le volume soit réduit à environ $10 \mathrm{~cm}^{3}$.

On y ajoute $2 \mathrm{~cm}^{3}$ de solution saturée d'oxalate d'ammonium et on mélange bien à l'aide d'une baguette en verre pour faciliter la précipitation du calcium. Après une demi-heure de repos, on centrifuge le liquide pour séparer le précipité d'oxalate de calcium et, ensuite, on verse le liquide surnageant, en renversant le tube.

Le précipité est lavé prudemment trois fois avec de l'eau 
distillée bouillante (en versant chaque fois $5 \mathrm{~cm}^{3}$ d'eau le long des parois du tube et en mélangeant le précipité) par centrifugation.

Le précipité séparé après le dernier lavage est dissout à chaud avec $5 \mathrm{~cm}^{3}$ d'acide sulfurique environ normal et dans cette solution l'acide oxalique mis en liberté est dosé à l'aide d'une solution centième normale de permanganate de potassium.

\section{LES PROTIDES EN BLOC}

Le papier, imbibé avec du lait et dont on a séparé, comme il a été indiqué précédemment, d'abord les substances solubles en éther sulfurique (les lipides) et ensuite les substances solubles dans la solution centième normale d'acide acétique (glucides et sels), est desséché à l'étuve à $100^{\circ}-103^{\circ}$, jusqu'à poids constant.

Après cela il est introduit dans une fiole en verre et laissé avce celle-ci un quart d'heure au dessiccateur.

Finalement, il est pesé avec la fiole. Ce dernier poids, diminué du poids initial (fiole + papier), donne la quantité des substances protéiques de la prise d'essai de lait.

\section{LA CASÉINE}

Vu l'état physique sous lequel se trouvent les matières protéiques du lait absorbé dans le papier à filtrer, nous avons pensé que le seul moyen qui pourrait nous faire cormaître la part, qui de l'ensemble des matières protéiques, revient à la caséine, serait le dosage de l'ácide phosphorique. Cela, pour cette raison que, de toutes les matières protéiques qui se trouvent dans le lait, la seule qui contient du phosphore, e'est la caséine.

Admettons, avec la plupart des auteurs, une teneur en phosphore de 0,8 pour la caséine du lait de vache, et 0,7 pour la caséine du lait de femme. Cela admis, voici comment on arrive à doser la caséine :

Le papier contenant les substances protéiques est introduit dans un creuset en quartz et humecté avec une dizaine de gouttes d'une solution d'acétate de magnésium à $20 \%$. On évapore au bain-marie et, ensuite, on incinère dans un four jusqu'à l'obtention des cendres blanches.

Des pertes en phosphore ne sont pas à craindre si l'on a ajouté une quantité suffisante d'acétate de magnésium.

Les cendres sont dissoutes dans $10 \mathrm{~cm}^{3}$ d'acide sulfurique à $20 \%$ et, dans cette solution, on dose l'acide phosphorique par la méthode de Copaux, lorsqu'il s'agit du lait de vache dont la teneur en caséine est assez élevée, ou par la méthode colorimétrique s'il s'agit du lait de femme, moins riche en caséine.

Nous croyons nécessaire à présent de décrire la manière dont nous avons utilisé, dans le cas du lait, les deux méthodes ci-dessus indiquées pour le dosage de l'acide phosphorique. 


\section{MÉTHOdE dE COPAUX}

Le principe de cette méthode est le suivant : Lorsqu'on agite une solution contenant de l'acide phosphorique (solution convenablement acidulée par un acide minéral) avec de l'éther exempt d'aleool et avec une solution de molybdate de sodium, il se produit un complexe liquide jaune, lequel, en vertu de sa grande densité, se sépare assez facilement du reste de la solution.

Pour mesurer le liquide ainsi séparé et la quantité d'acide phosphorique correspondante, nous avons procédé, en nous inspirant des travaux de Mme HinglaIs (1), de la manière suivante: Nous avons fait construire des ampoules en verre ayant des dimensions convenables, afin qu'elles puissent s'adapter au centrifugeur électrique dont nous disposions (Jouan). La longueur et le volume d'une telle ampoule sont de 9,5 à $10 \mathrm{~cm}$. et de $20-23 \mathrm{~cm}^{3}$. La douille, ayant un diamètre intérieur d'environ $0 \mathrm{~cm}$. 18 et une longueur d'environ $4 \mathrm{~cm}$. 2, est divisêe en 50 parties égales, de sorte que le volume compris entre deux divisions successives est d'environ d'un quatre-soixantième de centimètre cube.

A l'aide d'une loupe on peut enfin apprécier la moitié d'une division. Par conséquent nous pouvons considérer la douille divisée en 100 parties. Ces ampoules ont été étalonnées à l'aide d'une solution de phosphate acide d'ammonium, dont le titre en phosphore était de $0 \mathrm{gr}$. 09722 par litre.

L'étalonnage se fait de la façon suivante : Dans l'ampoule on introduit successivement :

$5 \mathrm{~cm}^{3}$ d'acide sulfurique à $20 \%$.

$\mathrm{n} \mathrm{em}^{3}$ de la solution titrée de phosphate d'ammonium.

$5-\mathrm{n} \mathrm{cm}^{3}$ de l'eau distillée.

$4 \mathrm{~cm}^{3}$ d'éther exempt d'alcool (2).

$2 \mathrm{~cm}^{3} 5$ de solution de molybdate de sodium, $140 \mathrm{gr}$. par litre.

On ferme l'ampoule avec le pouce et on l'agite vigoureusement, en la renversant plusieurs fois. On ajoute encore $2 \mathrm{~cm}^{3} 5$ de la solution de molybdate de sodium, en procédant exactement comme avant. Après 2 minutes de centrifugation, au centrifugeur électrique, le liquide jaune est nettement séparé.

Dans le tableau qui suit, nous donnons les nombres des divisions

(1) Mme H. Hingrais. Sur l'applieation de la méthode de M. Copaux au dosage de petites quantités de phosphore dans les tissus. Bulletin de la Société de Chimie biologique, t. IX, p. $540,1927$.

(2) L'éther est débarrassé d'alcool de la manière suivante :

On introduit dans un entonnoir à séparation, ayant la capacité de $1.000 \mathrm{~cm}^{3}$, un mélange de $144 \mathrm{~cm}^{3} \mathrm{~d}^{3}$ eau distillée et $56 \mathrm{~cm}^{3}$ d'acide sulfurique concentré $(\mathrm{D}=1.833)$, et après refroidissement sous un courant d'eau, $200 \mathrm{~cm}^{3} \mathrm{~d}^{\prime} e ́ t h e r$. Le mélange eșt agité vigoureusement plusieurs fois et ensuite est laissé au repos une heure. Après ce laps de temps, l'éther est séparé par déeantation. 
occupées par le liquide jaune séparé, en regard des quantités correspondantes de phosphore exprimées en milligrammes :

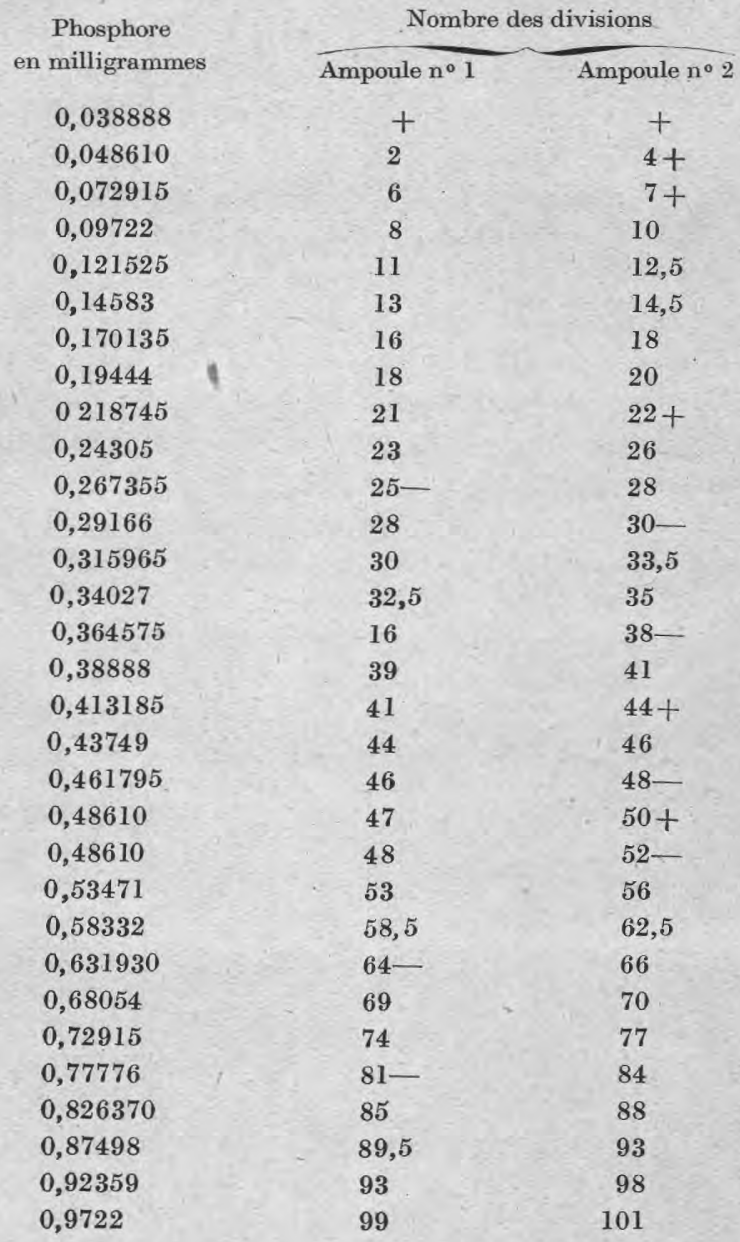

Voici maintenant comment on fait le dosage de l'acide phosphorique correspondant à la caséine incinérée : On introduit dans l'ampoule

$5 \mathrm{~cm}^{3}$ d'eau distillée.

$5 \mathrm{~cm}^{3}$ de la solution contenant l'acide sulfurique.

$4 \mathrm{~cm}^{3}$ d'éther exempt d'alcool, et enfin

$5 \mathrm{~cm}^{3}$ de la solution de molybdate de sodium en deux reprises, en procédant exactement comme il a été indiqué auparavant.

Dans le tableau ei-dessus nous donnons, en regard des chiffres 
représentant le nombre des divisions du liquide séparé, les quantités correspondantes de phosphore, exprimées en milligrammes.

\section{LA MÉTHODE COLORIMÉTRIQUE}

Dans $5 \mathrm{~cm}^{3}$ de la solution acide, contenant l'acide phosphorique, on introduit : $2 \mathrm{~cm}^{3}$ de lessive de soude à $40 \%$ et après refroidissement :

$2 \mathrm{~cm}^{3}$ d'une solution de molybdate d'ammonium (1).

$1 \mathrm{~cm}^{3}$ de solution de sulfite de sodium $20 \%$ (récemment préparée).

$1 \mathrm{~cm}^{3}$ de solution d'hydroquinone $0,5 \%$ (2).

Simultanément, dans un tube témoin, on introduit une quantité convenable d'une solution d'acide phosphorique, dont la concentration est connue et ensuite les mêmes réactifs que précédemment. (Il faut avoir soin que les deux tubes contiennent le même volume de liquide.)

On les agite pendant quelques instants et, après une demi-heure, on compare au colorimètre les intensités des couleurs bleues apparues.

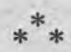

Dans le tableau qui suit, sont exposés les résultats des analyses faites sur une dizaine d'échantillons de lait de vache et de femme:

\begin{tabular}{|c|c|c|c|c|c|c|c|}
\hline & Prise d'essai & Résidu sec & Graisse & Lactose & Caséine & $\begin{array}{c}\text { Albumine }+ \\
\text { Globuline }\end{array}$ & Chlorures \\
\hline & 4 & 138,44 & 46,72 & 44,60 & 19,75 & 8,09 & - \\
\hline & 4,5 & 128,40 & 38,10 & 43,70 & 27,00 & 6,10 & 1,30 \\
\hline & 5 & 107,12 & 17,12 & 41,58 & 28,75 & 4,29 & 1,81 \\
\hline Vache. & 2,5 & 148,46 & 50,18 & 46,06 & 35,41 & 3,11 & 1,17 \\
\hline & 2,5 & 121,92 & 34,20 & 46,80 & 22,48 & 7,80 & 0,52 \\
\hline & 4 & 117,20 & 19,92 & 44,00 & 20,90 & 15,40 & - \\
\hline & 3 & 129,30 & 46,30 & 42,72 & 22,28 & 4,22 & 0,92 \\
\hline \multirow{3}{*}{ Femme } & 5 & 132,30 & 35,01 & 69,00 & 2,18 & 7,92 & - \\
\hline & 1 & 128,10 & 40,20 & 68,80 & 2,31 & 3,99 & - \\
\hline & 2,1 & 137,19 & 48,38 & 73,11 & 2,99 & 4,04 & - \\
\hline
\end{tabular}

(1) On dissout 25 grammes de molybdate d'ammonium dans $300 \mathrm{~cm}^{3}$ d'eau distillée, on filtre et, à cette solution, on ajoute un mélange refroidi de $25 \mathrm{~cm}^{3}$ d'acide sulfurique concentré et $125 \mathrm{~cm}^{3}$ d'eau.

(2) La conservation de cette solution est assurée en lui ajoutant une goutte d'acide sulfurique concentré. 